\title{
Comparison of Three Different Antibiotic Protocols Used as Prostate Biopsy Prophylaxis in Terms of Infectious Complications
}

\author{
(1) Aşkın Eroğlư ${ }^{1}$ ( ) Şahin Kılıç² \\ ${ }^{1}$ Başkent University Faculty of Medicine, Department of Urology, Izmir, Turkey \\ 2 Fethiye State Hospital, Clinic of Urology, Muğla, Turkey
}

\begin{abstract}
Objective: Development of urinary infection and sepsis following prostate biopsy procedure is the main problem. Despite use of antibiotic prophylaxis, which becomes a routine procedure in prostate biopsy, urinary infection and sepsis may significantly be observed. Furthermore, there is no consensus in terms of the type and duration of prophylaxis. This study aimed to compare the incidence of infectious complications, which developed during 3 different prophylaxis protocols used in our institution.

Materials and Methods: Four hundred and eighteen prostate biopsy procedures performed in our institution between 2010 and 2017 were evaluated retrospectively. Patients were divided into 3 groups based on their prophylactic antibiotic protocols. First group patients ( $n=136)$ were given ciprofloxacin and gentamicin $80 \mathrm{mg}$. Second group patients $(n=180)$ were given cefpodoxime and gentamicin $80 \mathrm{mg}$. Third group patients ( $n=102)$ were given cefpodoxime and gentamicin $160 \mathrm{mg}$. Three groups were compared in terms of post-biopsy infectious complications. SPSS 20 statistical program was used for data evaluation.

Results: There was no statistically significant difference between the groups in terms of age, prostate specific antigen level and prostate volume. Urinary tract infection following the prostate biopsy was seen in 11 (8.1\%) patients in group 1, 8 (4.4\%) patients in group 2 and, 2 (1.9\%) patients in group 3 . Five of these patients were hospitalized due to deterioration of their overall health status and fever. When 3 groups were statistically compared, infection incidence was significantly different between the first and the third group $(p<0.05)$.

Conclusion: Bacterial resistance against fluoroquinolones is particularly increasing day by day. Fluoroquinolone resistance in Escherichia coli strains in our country is reported between $39-63 \%$. In this study, infectious complications were found to be less in cefpodoxime plus gentamicin 160 mg prophylaxis, when compared to ciprofloxacin plus gentamicin $80 \mathrm{mg}$. Replacing quinolones with cefpodoxime and gentamicin in the antibiotic prophylaxis protocols used before prostate biopsy can minimize risk of infection.
\end{abstract}

Keywords: Prophylactic antibiotic, prostate biopsy, urinary tract infection

\section{Introduction}

Prostate cancer is the most common type of male cancer in developed countries as a result of increase in life expectancy (1). The standard diagnostic method of prostat cancer is transrectal ultrasound (TRUS) guided prostate biopsy (2). Although complications such as hematuria and hematospermia following prostate biopsy are common, they recover spontaneously. However, severe complications such as febrile and non-febrile urinary tract infection, epididymitis, acute prostatitis and sepsis may also be observed $(3,4)$. Antibiotic prophylaxis before the biopsy procedure in routine practice, can minimize these severe infectious complications. However, incidence of postbiopsy urinary infection and sepsis is increasing due to increase in bacterial antibiotic resistance (4). Furthermore, there is no consensus in terms of the type and duration of prophylaxis. This study aimed to compare the incidence of infectious complications, which developed during 3 different prophylaxis protocols used in our institution for the patients undergoing TRUS-guided prostate biopsy.

Cite this article as: Eroğlu A, Kılıç Ş. Comparison of Three Different Antibiotic Protocols Used as Prostate Biopsy Prophylaxis in Terms of Infectious Complications. Bull Urooncol 2020;19(2):56-59 


\section{Materials and Methods}

Four hundred and eighteen patients, who underwent TRUSguided prostate biopsy in our institution between 2010 and 2017, due to elevated prostate specific antigen (PSA) level and suspicion of prostate cancer following digital rectal examination were retrospectively evaluated.

\section{Selection and Description of Participants}

Re-biopsied patients and patients with any systemic chronic or immunosuppressive disease (Diabetes Mellitus, congestive heart failure, chronic renal failure, chronic liver failure, etc.) were excluded. Patients, who underwent standard 12-core prostate biopsy, were divided into 3 groups based on their prophylactic antibiotic protocols. First groups patients $(n=136)$ received 5-day course oral ciprofloxacin 500 mg, which started one day before the scheduled biopsy, and a single dose gentamicin $80 \mathrm{mg}$, which was administered intramuscularly 30 minutes before the procedure. Second group patients $(n=180)$ received 5 -day course oral cefpodoxime $200 \mathrm{mg}$ (oral $3^{\text {rd }}$ generation cephalosporin), which started one day before the scheduled biopsy, and a single dose gentamicin $80 \mathrm{mg}$, which was administered intramuscularly 30 minutes before the procedure. Third group patients $(n=102)$ received 5-day course oral cefpodoxime 200 mg, which started one day before the scheduled biopsy, and a single dose gentamicin $160 \mathrm{mg}$, which was administered intramuscularly 30 minutes before the procedure.

\section{Technical Information}

General Electric Healthcare, LOGIQ C2 ultrasound machine and biplane rectal probe were used during the biopsy procedure. Rectal enema was applied to the patients 2 hours before the biopsy for colon cleansing. TRUS-guided periprostatic block was applied to the patients with $2 \%$ prilocaine (CitanestR) before the biopsy for local anesthesia. All procedures, including the biopsy, were performed while the patient was in left lateral decubitus position with the knee and hip at flexion. Before the biopsy, the prostate was examined at the transvers and sagittal planes and the zonal anatomy and the ultrasonographic characteristics of the tissue were examined from the base to the apex of the prostate. Biopsy was performed with an 18-gauge, $22-25 \mathrm{~cm}$ biopsy needle and a biopsy gun with a suitable attachment. Patients were informed about potential complications in detail. First post-biopsy emergency admissions or admissions to the institution within the first month were evaluated in terms of infectious complications. Sepsis definition was based on the 2001 International Sepsis Definitions Conference Criteria (5).

\section{Statistical Analysis}

Three groups were compared in terms of post-biopsy infectious complications. SPPS 20 (IBM Corp. Released 2011. IBM SPSS Statisticsfor Windows, Version20.0. Armonk, NY:IBM Corp.) statistical program was used for the evaluation of data. Median \pm standard deviation, percentage and frequency values were used for the variables. In addition to this, Levene's test was used to assess equality of variances, as a precondition of parametric tests. Assumption of normality was tested by Shapiro-Wilk test.
Unidirectional variance analysis was used for comparing 3 or more groups, and when preconditions for parametric test were not met with the multiple comparison test Tukey Honestly Significant Difference, Kruskal Wallis and multiple comparison test, Bonferroni-Dunn, were used. Relationship between categorical variables was analyzed with Fisher's exact test and chi-square test. When expected frequencies were smaller than 20\%, Monte Carlo Simulation Method was used to include these frequencies in the analysis. For statistical significance, $p<0.05$ and $p<0.01$ were considered as the thresholds. .

\section{Results}

Mean age of patients in group 1, 2 and 3 was calculated as 65.7, 67.2 and 66.8 years, respectively and there was no statistically significant difference between the groups in terms of mean age $(p=0.31)$. Mean PSA level of patients in group 1, 2 and 3 was calculated as 9.99, 19.97 and $14.26 \mathrm{ng} / \mathrm{dL}$, respectively and there was no statistically significant difference between the groups in terms of PSA levels $(p=0.056)$. Mean prostate volume for the patients in group 1, 2 and 3 was calculated as 38.47, 42.16 and $44.27 \mathrm{~mL}$, respectively and there was no statistically significant difference between the groups in terms of mean prostate volume $(p=0.274)$. Urinary tract infection associated with biopsy was confirmed with urine culture. It developed in $11(8.1 \%)$ of 136 patients in the first group, 8 (4.4\%) of 180 patients in the second group and $2(1.9 \%)$ of 102 patients in the third group. Twenty one (5.02\%) patients out of a total of 418 patients developed urinary tract infection. Five (1.19\%) of these patients were hospitalized due to deterioration of their overall health status and fever. E.coli colonization was identified in the urine culture of 19 patients and Klebsiella spp. in 2 patients. Rate of resistance of E.coli against fluoroquinolone, aminoglycoside and $3^{\text {rd }}$ generation cephalosporin was identified in the antibiograms $80 \%, 42 \%$ and $28 \%$, respectively. When 3 prophylaxis groups were compared between each other in terms of post-biopsy infectious complications, no statistically significant difference was found between group 2 and the other groups. However, infectious complications were observed statistically less in group 3 , when compared to group $1(p<0.05)$.

\section{Discussion}

Prostate biopsy is an invasive urological intervention commonly applied in the present urology practice for diagnosis of prostate cancer. It may be applied with transperineal and transrectal approaches with similar complication rates (6). The most common complication following prostate biopsy is hematospermia (37.4\%)(4). Post-biopsy infectious complications are generally reported following $5-7 \%$ of procedures (7). The most common type of infectious complication is symptomatic urinary infection, which manifests without fever or with mild fever mainly associated with lower urinary tract symptoms (8). Febrile urinary tract infection requiring hospitalization and parenteral treatment, bacteremia and sepsis were reported following $0.64-3 \%$ of procedures $(7,8,9)$.

Most commonly isolated pathogen in symptomatic infections after prostate biopsy is E.coli $(7,10)$. Although there is consensus for use of prophylactic antibiotic, it is not clear yet which 
antibiotic to use and for how long. Furthermore, antimicrobial resistance rates vary from country to country (11). Concentration of the drug selected for antibiotic prophylaxis, in the prostatic parenchyma, periprostatic spaces and blood circulation, should not exceed the minimum inhibitor concentration of the uropathogens diffused during the procedure. Besides, drug of choice must be safe, cheap, easy to administer and efficacious against both Gram-negative and Gram-positive bacteria (12). In general, fluoroquinolones are mostly preferred for antibiotic prophylaxis. However, in selection of antibiotics, it is recommended to consider the regional and local antibiotic resistance patterns (4). In our country, there is fluoroquinolone resistance in E.coli strains, which is steadily increasing and has reached to $63 \%$ in the recent years (13). In general, while resistance against cephalosporins in E.coli strains in our country varies between $12 \%$ and $42.9 \%$, resistance against gentamicin is reported between $8 \%$ and $24 \%(13,14)$. Although there are regional differences, infectious complication rate in prostate biopsy will steadily increase in parallel to increasing rate of bacterial resistance (4). This requires the need for different prophylaxis protocols including single or combined antibiotics. There are several different prophylaxis protocols in the literature $(7,10,15)$. Furthermore, targeted antimicrobial prophylaxis based on measuring regional E.coli resistance rates by taking pre-biopsy rectal culture is also reported (10). BaldisseraAradasa et al. (16) reported a significant reduction in infectious complication rates and treatment cost of these complications with the use of targeted antimicrobial prophylaxis. However, in a study investigating 3953 targeted prophylaxis treatments in 15236 prostate biopsies, it was found that targeted prophylaxis did not have a significant effect in preventing sepsis. Fluoroquinolone resistance of E.coli was reported as $28.5 \%$ (10).

There was nearly two times difference between group 1 and 2 in terms of infection after biopsy, but the difference did not reach statistically significance. We think that antibiotic prophylaxis could change in favor of cefpodoxime by performing studies with larger sample size. A statistically significant difference among the group 1 and group 3 in terms of infection rates after biopsy suggested that increased dose of gentamicin $(160 \mathrm{mg}$ ) in group 3 provided an additional contribution to cefpodoxime in infection prophylaxis.

\section{Study Limitations}

The limitations of this study were its retrospective design and lack of group including ciprofloxacin + gentamicin with escalating doses. If such a group had been included in the study, the relationship between increased gentamicin dose and infection rates could have been made clearer.

\section{Conclusions}

In our study, taking into account fluoroquinolone resistance rates for E.coli strains in our country, we evaluated the efficacy of cefpodoxime and gentamicin prophylaxis. Although urinary infection rates in each of our 3 prophylaxis groups were in general similar to the results reported in the literature, there was a significant decrease in infection rates in cefpodoxime and gentamicin groups, with the increasing dose of gentamicin.
The limitation of our study was that cefpodoxime resistance was not checked in routine culture-antibiogram practice of our institution and no comment was provided on the resistance rates of our patients against cefpodoxime. Cefpodoxime is safe and easy to administer for prostate biopsy prophylaxis and is efficacious against both Gram-negative and Gram-positive bacteria, however, it is disadvantageous in terms of cost when compared to fluoroquinolones. Gentamicin has the challenge of parenteral administration. However, decrease in infectious complication rates with the use of cefpodoxime and gentamicin prophylaxis will in its turn reduce the cost of treatment for complications. Steady increase in fluoroquinolone resistance rate in our country and the results of our study support that cefpodoxime and gentamicin can be an alternative prophylaxis protocol in prostate biopsy. Randomized controlled studies to be conducted in larger patient groups including costeffectiveness analysis will further shed a light upon the choice of prophylactic antibiotic.

\section{Acknowledgements}

Publication: This study was presented in the $13^{\text {th }}$ International Urooncology Congress.

Contribution: There is not any other contributors who may not be listed as authors.

Conflict of Interest: No conflict of interest was declared by the authors.

Financial Disclosure: The authors declared that this study received no financial support.

\section{Ethics}

Ethics Committee Approval: Retrospective study.

Informed Consent: Retrospective study.

Peer-review: Externally peer-reviewed.

Authorship Contributions

Concept: A.E., Design: Ş.K., Data Collection or Processing: A.E., Ş.K. Analysis or Interpretation: A.E., Ş.K., Literature Search: A.E., Ş.K., Writing: A.E., Ş.K.

\section{References}

1. Ferlay J, Soerjomataram I, Dikshit R, et al. Cancer incidence and mortality worldwide: sources, methods and major patterns in GLOBOCAN 2012. Int J Cancer 2015;136:359-386.

2. Hodge KK, McNeal JE, Stamey TA. Ultrasound guided transrectal core biopsies of the palpable abnormal prostate. J Urol 1989;142:66-70.

3. Loeb S, Vellekoop A, Ahmed HU, et al. Systematic review of complications of prostate biopsy. Eur Urol 2013;64:876-892.

4. http://uroweb.org/guideline/prostate-cancer/\#5

5. Levy MM, Fink MP, Marshall JC, et al. 2001 CCM/ESICM/ACCP/ATS/ SIS International Sepsis Definitions Conference. Intensive Care Med 2003;29:530-538.

6. Xue J, Qin Z, Cai $\mathrm{H}$, et al. Comparison between transrectal and transperineal prostate biopsy for detection of prostate cancer: a meta-analysis and trial sequential analysis. Oncotarget 2017;8:23322-23336

7. Fontana $\mathrm{M}$, Boeri L, Montanari E. Update on techniquest prevent infections associated with prostate needle biopsy. Curr Opin Urol 2018;28:392-397. 
8. Hamarat MB, Tarhan F, Horuz R, et al. Infective complications in patients after transrectal ultrasound-guided prostate biopsy and the role of ciprofloxacin resistant Escherichia colicolonization in rectal flora. Turk J Urol 2017; 43:210-215.

9. Qi DZ, Lehman K, Dewan K, et al. Preoperative urine culture is unnecessary in asymptomatic men prior to prostate needle biopsy. Int Urol Nephrol 2018;50:21-24.

10. Jiang P, Liss MA, Szabo RJ. Targeted antimicrobial prophylaxis does not always prevent sepsis after transrectal prostate biopsy. J Urol 2018;200:361-368.

11. https://ecdc.europa.eu/sites/portal/files/documents/AMRsurveillance-EARS-Net-2017.pdf.

12. Wolf JS, Bennett CJ, Dmochowski RR, et al. Best practice policy statement on urologic surgery antimicrobial prophylaxis. J Urol 2008;179:1379-1390.
13. Yılmaz N, Ağuş N, Bayram A, et al. Antimicrobial susceptibilities of Escherichia coli isolates as agents of community-acquired urinary tractin fection (2008-2014). Turk J Urol 2016;42:32-36.

14. Arslan H, Azap OK, Ergönül O, et al. Urinary Tract Infection Study Group. Risk factors for ciprofloxacin resistance among Escherichia coli strains isolated from community-acquired urinary tract infections in Turkey. J Antimicrob Chemother 2005;56:914-918.

15. Elshal AM, Atwa AM, El Nahas AR, et al. Chemoprophylaxis during transrectal prostate needle biopsy: critical analysis through randomized clinical trial. World J Urol 2018;36:1845-1852.

16. Baldissera-Aradasa JV, Rodríguez-Villamila L, Blanco-Fernándeza R, et al. Rectal culture-directed antibiotic prophylaxis before transrectal prostate biopsy: Reduced infectious complications and health care costs. Actas Urol Esp 2018;42:465-472. 